\title{
Phonon spectroscopy measurements at amorphous films
}

\author{
J. Mebert ${ }^{1, \star}$, W. Eisenmenger ${ }^{2}$ \\ ${ }^{1}$ TÜV-Südwest, Nuclear Power and Radiation Protection Department, Raiffeisenstrasse 30, D-70794 Filderstadt Bonlanden, Germany \\ ${ }^{2}$ Physikalisches Institut Teil 1, Universität Stuttgart, Pfaffenwaldring 57, D-70569 Stuttgart, Germany
}

Received: 10 January 1994/Revised version: 2 March 1994

\begin{abstract}
Phonon spectroscopy measurements were used to examine the scattering of high frequency phonons $(300 \mathrm{GHz}-1 \mathrm{THz})$ in amorphous materials. The experiments were done with the use of time and frequency resolved measurements of the phonon transmission behaviour through amorphous single films of different thicknesses. The typical film thicknesses were of the order of $10 \mathrm{~nm}$. In contrast to the pure amorphous semiconductors $\mathrm{Si}$ and $\mathrm{Ge}$ our experiments show inelastic phonon scattering processes in the case of $\mathrm{SiO}_{2}$ and $\mathrm{Si}: \mathrm{H}$. This inelastic phonon scattering also occurs when the pure semiconductors $\mathrm{Si}$ and $\mathrm{Ge}$ are prepared in an $\mathrm{O}_{2}$ or $\mathrm{H}_{2}$ atmosphere, but is missing when the preparation process is done in an $\mathrm{N}_{2}$ atmosphere. In films of the pure semiconductors a-Si and a-Ge we only found evidence to elastic scattering processes. In further experiments at heated a-Si:H samples we could examine the atomical bonded hydrogen to be the center of the inelastic phonon scattering.
\end{abstract}

PACS: $61.42 .+\mathrm{h} ; 62.40 .+\mathrm{i} ;$ 68.60.Bs

\section{Introduction}

In the last years a lot of experiments were done to examine the phonon scattering processes in amorphous systems. Some of these experiments were measurements of the temperature dependence of the thermal conductivity. A disadvantage of such measurements is not to give the possibility to distinguish between elastic or inelastic phonon scattering processes. Such a possibility of distinction is given by phonon spectroscopy measurements.

First phonon spectroscopy measurements on evaporated amorphous $\mathrm{SiO}_{2}$ films were done by Dietsche and

* The measurements and investigations described in this work were done in time of preparing a thesis at: Physikalisches Institut Teil 1, Universität Stuttgart, Pfaffenwaldring 57, D-70569 Stuttgart, Germany
Kinder [4]. With the use of Sn-tunnel-junctions as phonon generator these measurements are limited to the frequency range below $300 \mathrm{GHz}$. A short time later Wolter and Horstmann [22] published their phonon spectroscopy measurements on phonon transmission through thermal oxide in the same frequency range. The experiments of Dietsche and Kinder gave strong evidence on the existence of inelastic phonon scattering processes in $a-\mathrm{SiO}_{2}$. This result could not be confirmed by Wolter and Horstmann. Thus the question about the phonon scattering processes in amorphous $\mathrm{SiO}_{2}$ was still open.

A strong motivation in this work was therefore to give additional information in the question of the phonon scattering processes in $a-\mathrm{SiO}_{2}$. A central point in our investigations was the extension of the accessible frequency range up to frequencies above $300 \mathrm{GHz}$. Additionaly we extended our measurements on several other amorphous materials such as on the pure amorphous semiconductors $a-\mathrm{Si}$ and $a$-Ge as well as on hydrogenand oxygen-prepared semiconductor films.

\section{Phonon spectroscopy measurements}

\section{A. Phonon transmission spectra}

Superconductive tunneling junctions can be used as phonon generator as well as phonon detector [5]. The energy spectrum of the relaxation phonons emitted by the generator junction has a sharp cutoff at the energy $e U-2 \Delta_{g}$, where $2 \Delta_{g}$ is the superconducting gap energy of the generator material and $U$ the DC voltage applied to the junction. Using special modulation techniques this sharp cutoff of the relaxation spectrum can be used to generate a quasimonochromatic phonon line. The frequency of this line is tunable with the applied DC voltage [14], [6].

To describe some typical characteristics of phonon transmission spectra we refer to Figs. 1 and 2. The rise in the detector signal at $285 \mathrm{GHz}$ is due to the threshold of phonon detection in the $\mathrm{Sn}$ detector. Other typical structures are the structure below the detector threshold 


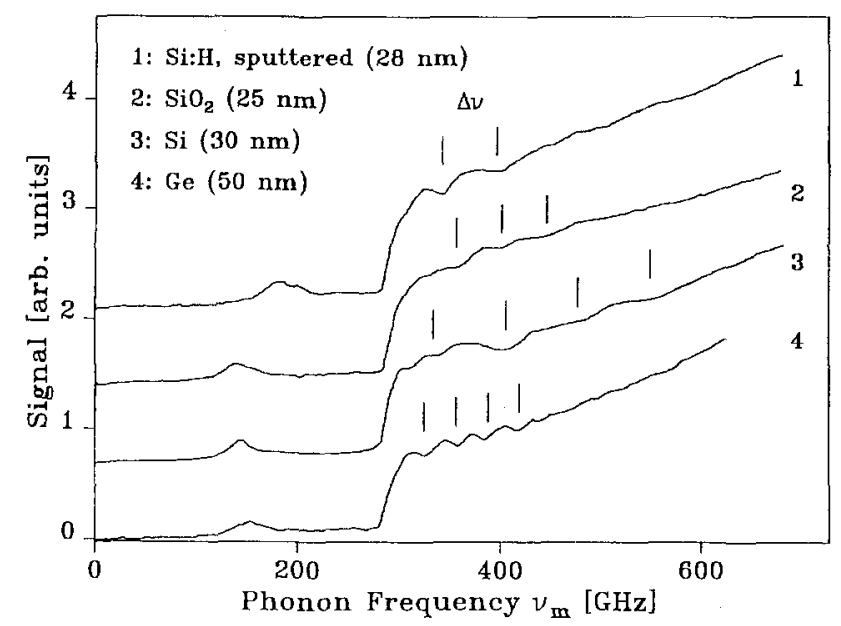

Fig. 1. Thickness-resonances in the phonon transmission spectra of amorphous films. Using the frequency-distance between two minima the sound velocity of TA phonons in the amorphous film can be evaluated

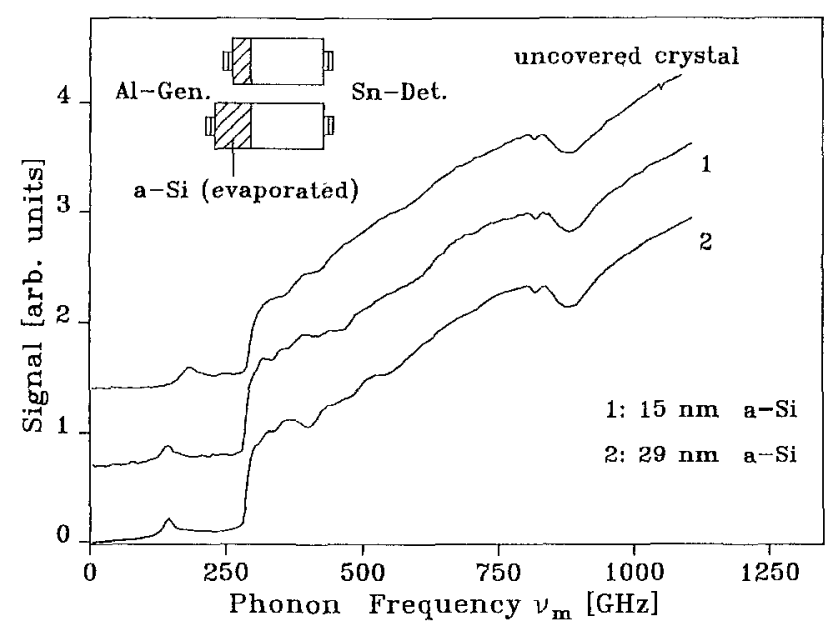

Fig. 2. Phonon transmission spectrum of $a$-Si films. The curves 1 and 2 show the measurements of a "double crystal experiment" with $a$-Si films of $15 \mathrm{~nm}$ and $29 \mathrm{~nm}$ thickness. The upper curve is the typical transmission spectrum of an uncovered sample

and the deep absorption structure at $875 \mathrm{GHz}$. The structure below the detector threshold is called "thermal precursor" and arises from the thermal current in the Algenerator [6]. The absorption structure at $875 \mathrm{GHz}$ is due to a vibrational mode of the interstitial oxygen in the $\mathrm{Si}$ crystal [8].

For further more detailed discussion of the principles of phonon spectroscopy measurements we refer to the basic work of Eisenmenger and Dayem ([5], [6]) and to that of Kinder [14].

\section{B. Experimental arrangement}

In our experiments on phonon scattering in amorphous systems we used an arrangement described in the following passage. The specific arrangement of each measurement is also shown in the insert of the respective figure.
To carry the amorphous films we used crystalline $\mathrm{Si}$ substrates doped with oxygen at a concentration of $3.8 \cdot 10^{17} \mathrm{~cm}^{-3}$. The thickness of the substrates was $4 \mathrm{~mm}$ and their orientation axis was the $\langle 111\rangle$ direction in all measurements. The amorphous films were prepared on the polished surface of the carrier-crystal. This surface had an area of $14 \times 14 \mathrm{~cm}^{2}$. The typical film thickness was of the order of $10 \mathrm{~nm}$. In a separate preparation process we evaporated the $\mathrm{Al}$ and $\mathrm{Sn}$ tunnel junctions with a tunneling area of $1 \mathrm{~mm}^{2}$. The Al tunnel junction was used as phonon generator the Sn tunnel junction as phonon detector. In this arrangement the useful frequency range extends from $285 \mathrm{GHz}$ up to $3 \mathrm{THz}$. In most experiments the $\mathrm{Al}$ junction was evaporated on top of the amorphous film and the detector junction on the uncovered opposite surface of the carrier-crystal.

In the time resolved measurements the $\mathrm{Al}$ junction was replaced by a constantan heater with a resistance of $50 \Omega$. The thickness of the heater was $300 \AA$, the length $1 \mathrm{~mm}$ and the width $100 \mu \mathrm{m}$.

To eliminate surface effects our experiments were performed as "double crystal experiments". That means that the amorphous material is prepared in different thickness on top of two identical carrier-crystals. The evaporation of the phonon generators and detectors was done in a further step on both samples at the same time. Differences in the transmission spectra of such a "double crystal experiment" can therefore be attributed to the different thickness of the amorphous films. In this way they can be interpreted as resulting from the amorphous system. In the special arrangement with the phonon generator on top of the amorphous film the phonons are first transmitted through the amorphous film before they can interact with the carrier crystal. In this way the phonon spectrum emitted by the Al generator is directly modified by scattering processes in the amorphous system and can then be analysed via the absorption properties of typical resonances of the carrier-crystal.

\section{Sample preparation}

\section{A. Evaporated films}

The evaporated films were prepared in an automatic ultrahigh vacuum (UHV) evaporation system. The materials were evaporated by an electron beam gun. The starting pressure for all processes was in the range of $7-9 \cdot 10^{-9}$ mbar. In order to reach this value the system was heated for $12 \mathrm{~h}$ at temperatures of $120-140^{\circ} \mathrm{C}$.

A watercooled quartz crystal monitor was used to control the film thickness and the evaporation rate. The calibration of the quartz crystal monitor was done in the way described in Sect. IVA. The evaporation rate was $0.25 \mathrm{~nm} / \mathrm{s}$ in all cases.

The amorphous $\mathrm{Si}$ and $\mathrm{Ge}$ films prepared in an $\mathrm{O}_{2}$, $\mathrm{H}_{2}$ and $\mathrm{N}_{2}$ background gas atmosphere were also evaporated in the UHV evaporation system. The pressure of the background gas was controlled by a quadrupole mass spectrometer to a value of $5 \cdot 10^{-5}$ to $10^{-4} \mathrm{mbar}$. Under these conditions it is possible that $60 \%$ of all evaporated 
particles can react with gas molecules. Additionaly the recovering time is very small. Therefore there is a high probability of phyiscal inclusion of background gas molecules in the amorphous film. In this way it was possible to modify the gas concentration in the film by changing the gas pressure during the evaporation process.

\section{B. Thermally oxidized films}

In addition to our measurements at evaporated $\mathrm{SiO}_{2}$ films we also investigated $\mathrm{SiO}_{2}$ films prepared by thermal oxidation. Such films have a more homogeneous and a more dense structure than evaporated films. The preparation of the thermally oxidized samples was done at the "Max Planck Institut für Festkörperphysik" in Stuttgart. The carrier crystal was heated in a reaction chamber to a temperature of $1100^{\circ} \mathrm{C}$ in a constant $\mathrm{O}_{2}$-flux of $75 \mathrm{ml}$ $\mathrm{O}_{2} / \mathrm{min}$. In this process one surface of the crystal was covered whereas the other unprotected surface was oxidized. With known reaction rate the film thickness could be determined by the reaction time.

\section{PECVD-prepared films}

The fabrication of plasma-deposited Si and Ge films was done at the "Institut für Physikalische Elektronik" of the University of Stuttgart. For the deposition process the preparation chamber was evacuated to a residual gas pressure of $10^{-7} \mathrm{mbar}$. In the following procedure silane or germane was induced with a constant gas flux at a gas pressure of 0.1-1 mbar. The deposition process was supported by a DC glow discharge at a voltage of $1-1.5 \mathrm{kV}$. During the deposition process the chamber was kept at a temperature of $80^{\circ} \mathrm{C}$. The substrate temperature was $250^{\circ} \mathrm{C}$. Under these conditions the deposition rate was $0.25-0.27 \mathrm{~nm} / \mathrm{s}$.

The hydrogen content of such plasma deposited films is much enhanced compared to evaporated films. In our samples the $\mathrm{H}$-content was determined to $10-15$ at $\%$ by measurements of the optical Raman scattering.

\section{HF-sputtered films}

Some of our hydrogen doped Si films were prepared by HF-sputtering of $\mathrm{Si}$ in a $\mathrm{H}_{2}$ atmosphere of $5 \cdot 10^{-5}$ mbar. The preparation of these samples was also done at the "Max Planck Institut für Festkörperforschung" in Stuttgart. The pressure of the sputtering gas (Ar) was $10^{-3}$ mbar and the DC voltage was $2.2 \mathrm{kV}$. The H-concentration of the amorphous films was determined by Raman measurements to the value of $14 \mathrm{at} \%$.

\section{Experimental results}

\section{A. Sound velocities}

Using typical signal modifications in the phonon transmission spectra we could examine the sound velocities in the investigated systems in the following way.
- In a "double crystal experiment" we covered two carrier-crystals with the amorphous film. One sample was covered completely, the other remained uncovered with the exception of a small stripe.

- To determine the thickness of the evaporated film the sample with the small stripe was covered completely with $70 \mathrm{~nm} \mathrm{Al}$ in a second step. The thickness of the amorphous film stripe was then determined by the Tolansky Interference method. Covering with $\mathrm{Al}$ is important to avoid different phase changes by the reflexion of the light wave at the stripe and at the carrier surface.

The Tolansky plane we used had a reflectivity of $96 \%$. The resulting interference pattern was therefore very sharp. The interference pattern was photographed with the additional use of a microscope and evaluated with a photographical enlarger. In this way the thickness of the stripe could be determined with a precision of $2-3 \mathrm{~nm}$. To control our optically determined values we additionaly measured two of our samples with a mechanical profile scanner. In the range of measurement accuracy the values of the Tolansky measurement were confirmed.

At the completely covered sample we made measurements of the phonon transmission spectra (Fig. 1).

In the frequency range above the detector threshold a sequence of minima is observed. The frequency distance between two minima scales inversely with the thickness of the film. This behaviour is characteristic for thicknessresonances in the film. Since the generation rate of transverse phonons is much higher than the rate of longitudinal phonons these structures can be ascribed to TAphonons. In addition, the TA phonons are strongly focused in the $\langle 111\rangle$ direction due to anisotropic phonon propagation, whereas the LA phonons are only weakly focused. In the case of $\mathrm{Ge}$ the structures were more pronounced than in the case of $\mathrm{Si}$ or $\mathrm{SiO}_{2}$. This is due to the more homogenous structure of the $a$-Ge films.

As the complete film and the stripe were prepared at the same time and under the same conditions they had the same thickness. With known thickness the thicknessresonances can be used to determine the sound velocity of TA-phonons in the amorphous films as shown in Table 1.

The thickness values determined by the Tolansky measurements were also used to calibrate the quartz crystal monitor.

Table 1. Sound velocities $v_{T}$ and acustic impedances $Z$ of TA-phonons in amorphous films determined by phonon spectroscopy measurements

\begin{tabular}{llcc}
\hline Material & Preparation & $v_{T}[\mathrm{~km} / \mathrm{s}]$ & $\begin{array}{l}Z=\rho \cdot v_{T} \\
{\left[10^{5} \mathrm{gr} / \mathrm{cm}^{2} \mathrm{~s}\right]}\end{array}$ \\
\hline $\mathrm{Ge}$ & evaporated & $2.8 \pm 0.1$ & $11.2 \pm 0.40$ \\
$\mathrm{Si}$ & evaporated & $4.2 \pm 0.4$ & $9.0 \pm 1.0$ \\
$\mathrm{Si}: \mathrm{H}$ & sputtered & $3.0 \pm 0.3$ & $7.0 \pm 0.6$ \\
& PECVD & $4.0 \pm 0.4$ & $7.0 \pm 2.0$ \\
$\mathrm{SiO}_{2}$ & evaporated & $2.5 \pm 0.3$ & $4.0 \pm 1.0$ \\
\hline
\end{tabular}




\section{B. Phonon transmission measurements}

In the following measurements we compare spectra of the frequency dependent signal amplitudes of "double crystal experiments". For the quantitative comparison the signal amplitudes are adjusted to identical step height at the detector threshold. The detector threshold corresponds to the signal onset of the monochromatic phonon line at $285 \mathrm{GHz}$. This adjustment provides that both spectra are compared in their further course with respect to the intensity at $285 \mathrm{GHz}$. The adjustment does not mean that the absolute intensities of the phonon flux in both measurements is equal at this frequency. The adjustment or calibration at the detector threshold therefore is suited to detect differences in the phonon spectra above $285 \mathrm{GHz}$.

In our experimental arrangement described in Sect. IIB differences in the two phonon transmission spectra can only be affected by the different film thicknesses. They can therefore be attributed to the amorphous system.

Another frequency label which gives additional information is the depth of the absorption maximum of the vibrational excitation of the interstitial oxygen in the carrier substrate. This line depth is correlated to the intensity of the monochromatic phonon line at $875 \mathrm{GHz}$.

1. Evaporated a-Si. In Fig. 2 the phonon transmission spectra of a "double crystal experiment" of evaporated $a$-Si films are shown. The layer thicknesses were $15 \mathrm{~nm}$ (curve 1) and $29 \mathrm{~nm}$ (curve 2). For comparison the upper curve shows the phonon transmission spectrum of an uncovered sample. In this measurement the Al generators had reduced frequency gap. Therefore the distance between detector threshold and the thermal precursor is smaller than in the other measurements of the spectra with energy gap enhancement of the $\mathrm{Al}$ generator by oxygen background evaporation. There is no influence on the discussion.

The curves of the spectra coincide with the exception of the multiple resonance minima above the detector threshold. The absorption depth of the $875 \mathrm{GHz}$ resonance of the carrier-crystal does not depend on the thickness of the amorphous film and corresponds to that of an uncovered sample. The multiple resonance structure scales inversely with the film thickness. Therefore it can be ascribed to resonances in the amorphous film (Sect. IVA). The analogous structure in the spectrum of the uncovered sample is due to thickness resonances in the generator junction [7].

2. Evaporated $\mathrm{Ge}$. In the same way as Si films amorphous Ge films with thickness of some $10 \mathrm{~nm}$ do not affect the phonon transmission spectra besides film thickness resonances. This is shown in Fig. 3. The phonon transmission spectra of films with thicknesses of $30 \mathrm{~nm}$ and $60 \mathrm{~nm}$ have identical shape with the exception of the thickness resonance structures above the detector threshold. The spectra correspond to the spectrum of an uncovered sample. The reduced absorption depth of the $875 \mathrm{GHz}$ resonance of the carrier-crystal in the spectrum of the $60 \mathrm{~nm}$ layer is an artefact due to the increased modulation width in this measurement.

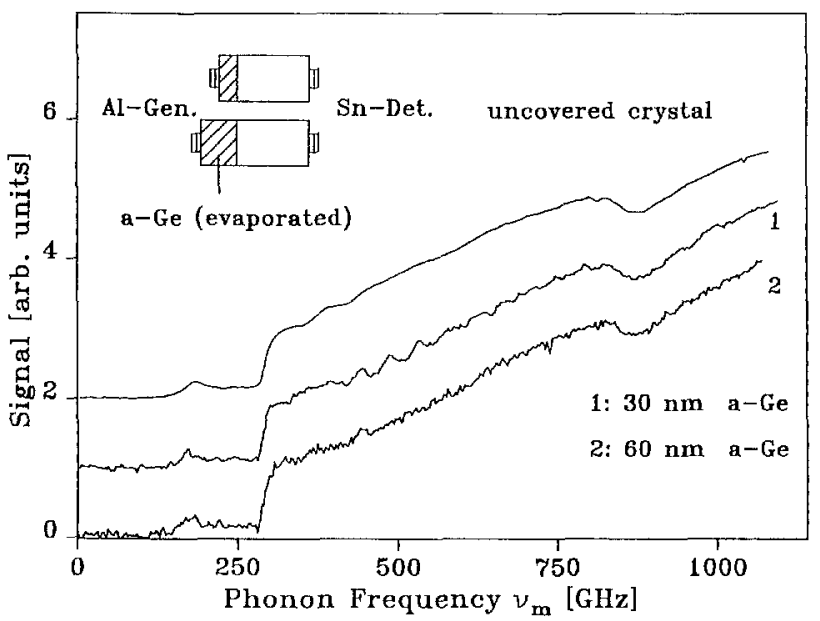

Fig. 3. Phonon transmission spectrum of $a$-Ge films. The film thicknesses are $30 \mathrm{~nm}$ (curve 1) and $60 \mathrm{~nm}$ (curve 2). The phonon transmission spectrum of the uncovered sample is identical with that of Fig. 2

3. Evaporated $\mathrm{SiO}_{2}$. Figure 4 shows the results of a "double crystal experiment" with $\mathrm{SiO}_{2}$ covered carrier crystals (curve 1 and 2). The film thicknesses were $19 \mathrm{~nm}$ and $38 \mathrm{~nm}$. The spectrum of the uncovered sample is identical to that in Fig. 2.

The both traces coincide up to a frequency of $400-$ $450 \mathrm{GHz}$. For frequencies above $400-450 \mathrm{GHz}$ the signal decreases with increasing film thickness of the $\mathrm{SiO}_{2}$ film. In the same way the absorption depth of the $875 \mathrm{GHz}$ resonance structure decreases with increasing film thickness. In the case of the $38 \mathrm{~nm} \mathrm{SiO}{ }_{2}$ film the absorption line vanishes rather completely.

Above $875 \mathrm{GHz}$ the gradient of the signal trace of the covered samples increases. That means that the shape of the spectrum is not characterised by a continously decreasing gradient. (In the range of the $875 \mathrm{GHz}$ resonance there can be recognized a positive change of the rise of

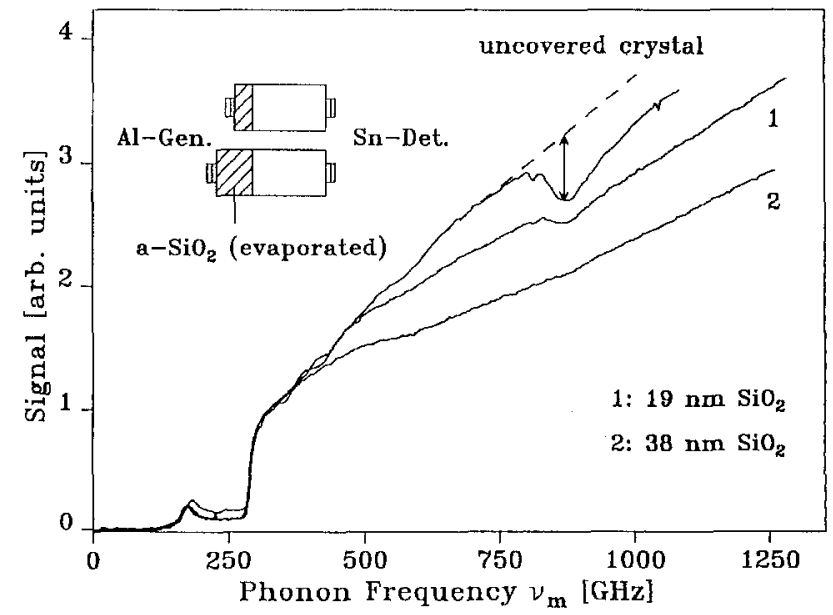

Fig. 4. Phonon transmission through evaporated $a-\mathrm{SiO}_{2}$ films. Trace 1 and 2 show the phonon transmission spectra through $\mathrm{SiO}_{2}$ films with thicknesses of $19 \mathrm{~nm}$ and $38 \mathrm{~nm}$. The upper trace shows the typical spectrum of an uncovered sample and is identical to that of Fig. 2. The arrow indicates the absorption depth of the vibrational resonance of the interstitial oxygen in the carrier crystal 
the course.) This behaviour implies an important hint on the scattering process in the $\mathrm{SiO}_{2}$ film (cp. Discussion).

In order to find out whether the signal reduction is an instrinsic influence of the $\mathrm{SiO}_{2}$ film or an influence induced by the phonon backscattering in the generator junction we made measurements at samples with the amorphous film placed between the carrier-crystal and the detector junction. The measurement is shown in Fig. 5. The signal trace is equivalent to that of a measurement with the amorphous film placed on top of the amorphous layer. Differences in the signal course can be recognized in the frequency range from $400 \mathrm{GHz}$ to $700 \mathrm{GHz}$ and in the absorption depth of the $875 \mathrm{GHz}$ resonance of the carrier-crystal. The differences in the frequency range from $400 \mathrm{GHz}$ to $700 \mathrm{GHz}$ are due to the thickness resonances of the $\mathrm{SiO}_{2}$ film in the case of the sample with the amorphous film between generator junction and carrier crystal. The difference in the absorption depth of the $875 \mathrm{GHz}$ resonance structure will be discussed later.

4. Thermal oxide. In order to investigate the influence of the network structure of the amorphous film on the phonon transmission spectrum we also made measurements on samples covered with thermal oxide. This oxide is much denser and has a more homogeneous network than evaporated $\mathrm{SiO}_{2}$.

In Fig. 6 the transmission spectra of two samples covered with thermally oxidized films with thicknesses of $30 \mathrm{~nm}$ and $60 \mathrm{~nm}$ are compared.

The spectra show the same characteristics as in the case of evaporated $\mathrm{SiO}_{2}$ with the exception of the traces coinciding up to $500 \mathrm{GHz}$. Furthermore the reduction of the depth of the $875 \mathrm{GHz}$ resonance structure is diminuished by the factor 3-4 compared with the reduction in an experiment with evaporated $\mathrm{SiO}_{2}$ films of the same thickness.

5. Oxygen prepared films. Figure 7 shows the results of "double crystal experiments" performed with amorphous $\mathrm{Si}$ and Ge films. The films have identical thicknesses, but

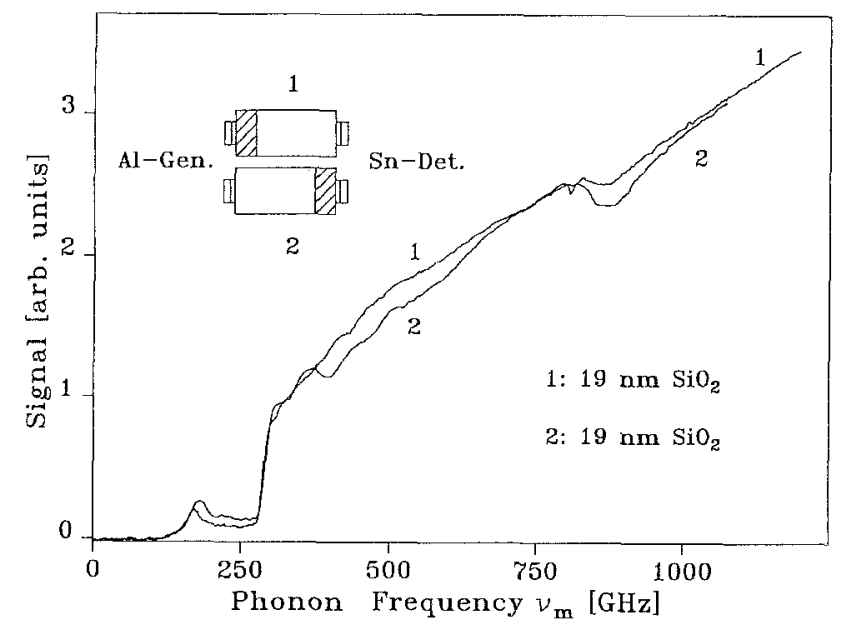

Fig. 5. Comparison of the signal reduction in the phonon transmission spectrum of $\mathrm{S} \mathrm{SiO}_{2}$ sample with the detector junction placed on top of the amorphous film and on the other hand with the generator junction placed on top of the amorphous film. The film thicknesses was $19 \mathrm{~nm}$ in both cases

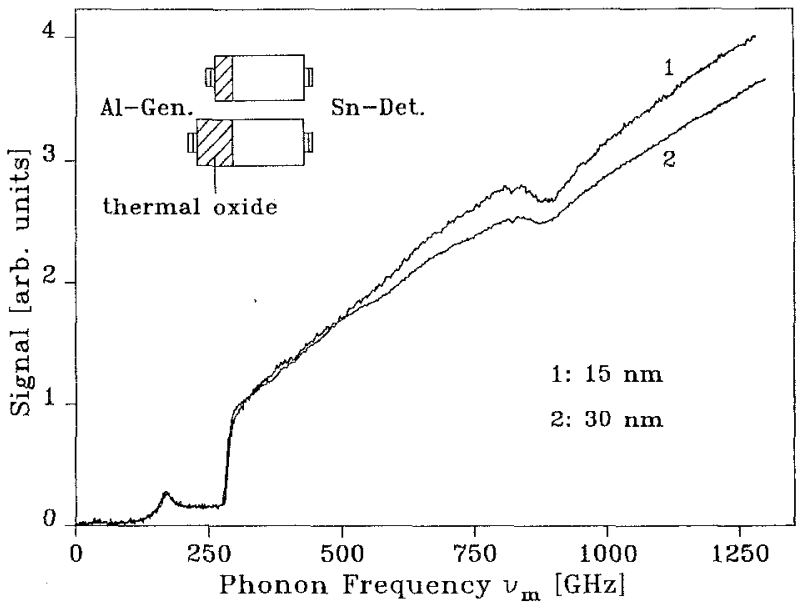

Fig. 6. Phonon transmission spectrum of two samples covered with thermal oxide

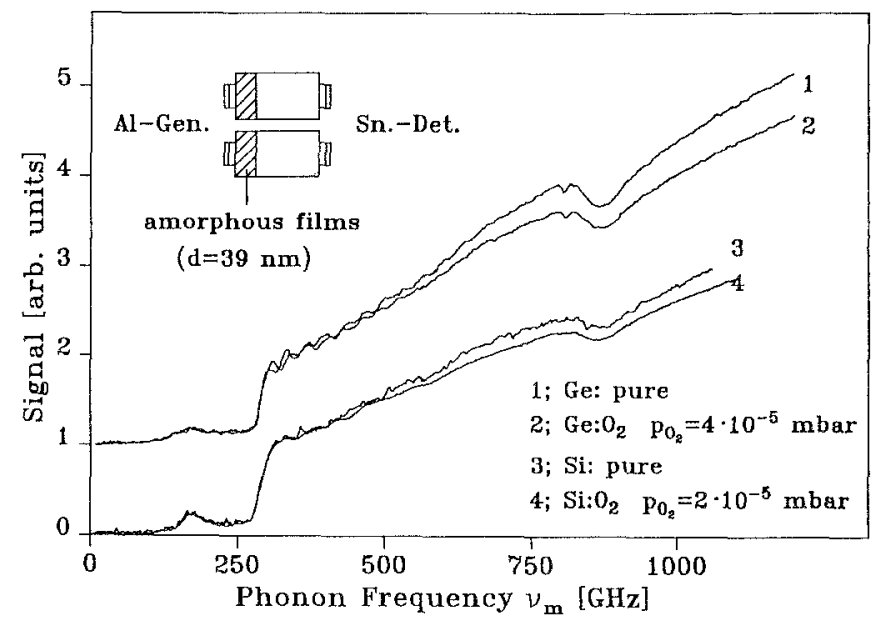

Fig. 7. Phonon transmission spectra of oxygen prepared amorphous semiconductors. The figure compares the spectra of films with identical thicknesses, one film prepared in an oxygen atmosphere of $2-4 \cdot 10^{-5}$ mbar, the other at zero oxygen partial pressure

one film each is prepared in an oxygen atmosphere. The signal in the phonon transmission spectrum of each oxygen prepared film is reduced with increasing phonon frequency compared to the spectrum of the pure amorphous semiconductor films. Further experiments with oxygen prepared $a$-Si and $a$-Ge films show the thickness dependence with the signal reduction above $400 \mathrm{GHz}$ and the reduction of the absorption depth of the $875 \mathrm{GHz}$ carrier crystal resonance structure.

6. Hydrogen prepared evaporated films. The measurements in Fig. 8 show the phonon transmission spectra of two $a$-Si films with thicknesses of $60 \mathrm{~nm}$ and $120 \mathrm{~nm}$ which were evaporated in a hydrogen atmosphere of $p_{H_{2}}=$ $4 \cdot 10^{-5}$ mbar. The difference between the traces is small due to the small hydrogen content of the films, but is significant with respect to a distinct signal reduction in the case of the $120 \mathrm{~nm}$ sample compared to the spectrum of the $60 \mathrm{~nm}$ sample. 


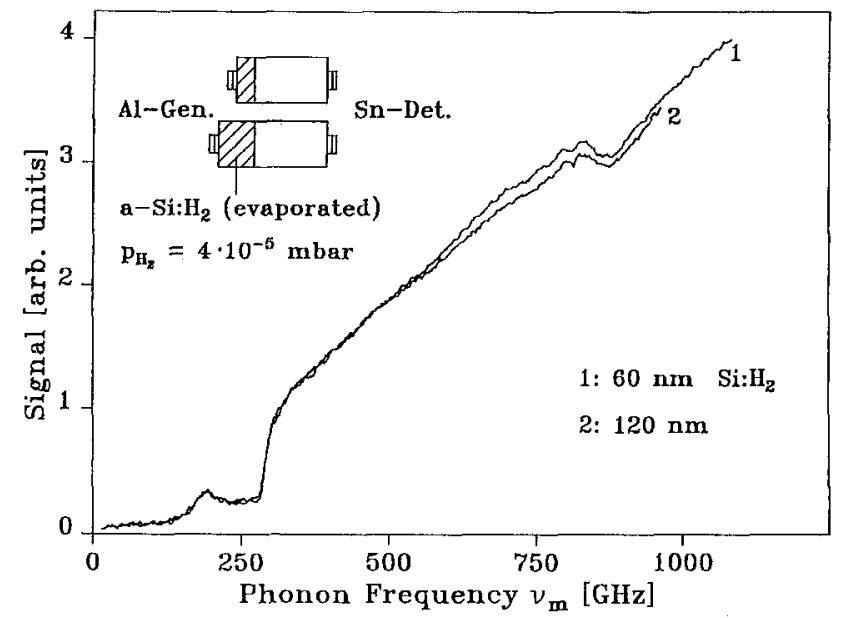

Fig. 8. Phonon transmission spectrum of a $60 \mathrm{~nm} \mathrm{Si:H}$ film (trace 1) compared with the spectrum of a $120 \mathrm{~nm}$ sample (trace 2). The films were evaporated in a hydrogen atmosphere of $p_{\mathrm{H}_{2}}=$ $4 \cdot 10^{-5} \mathrm{mbar}$

7. PECVD prepared $\mathrm{Si}: \mathrm{H}$. In order to investigate films with higher hydrogen content we also made measurements at PECVD prepared $\mathrm{Si}: \mathrm{H}$ films. The samples had an $\mathrm{H}$-content of some 10 at \%. In Fig. 9 the phonon transmission spectra of films with thicknesses of $40 \mathrm{~nm}, 60 \mathrm{~nm}$ and of $120 \mathrm{~nm}$ are compared. In these measurements we found again the thickness dependence of the signal reduction in the frequency range above $450 \mathrm{GHz}$. This signal reduction is accompanied by the reduction of the absorption depth of the $875 \mathrm{GHz}$ resonance structure of the carrier crystal.

8. Sputtered $\mathrm{Si}: \mathrm{H}$ films. Amorphous Si films containing hydrogen can also be prepared by $\mathrm{HF}$ sputtering. We are able to produce $\mathrm{Si}$ films with an hydrogen content of $14 \mathrm{at} \%$. In Fig. 10 the phonon transmission spectra of such films with thicknesses of $16 \mathrm{~nm}$ and $20 \mathrm{~nm}$ are compared.

According to the higher hydrogen content the signal reduction and the reduction of the absorption depth of

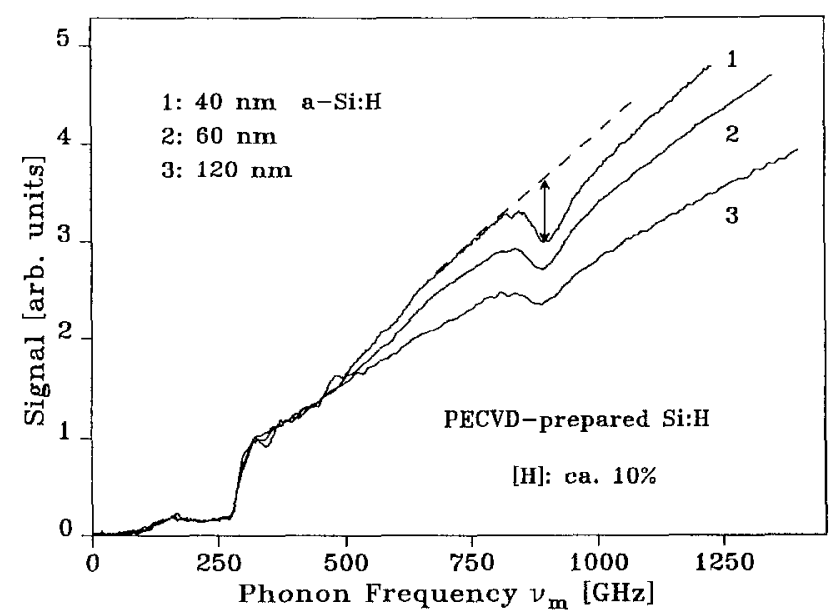

Fig. 9. Phonon transmission spectra of PECVD prepared Si films. The films had an $\mathrm{H}$-content of $10 \mathrm{at} \%$

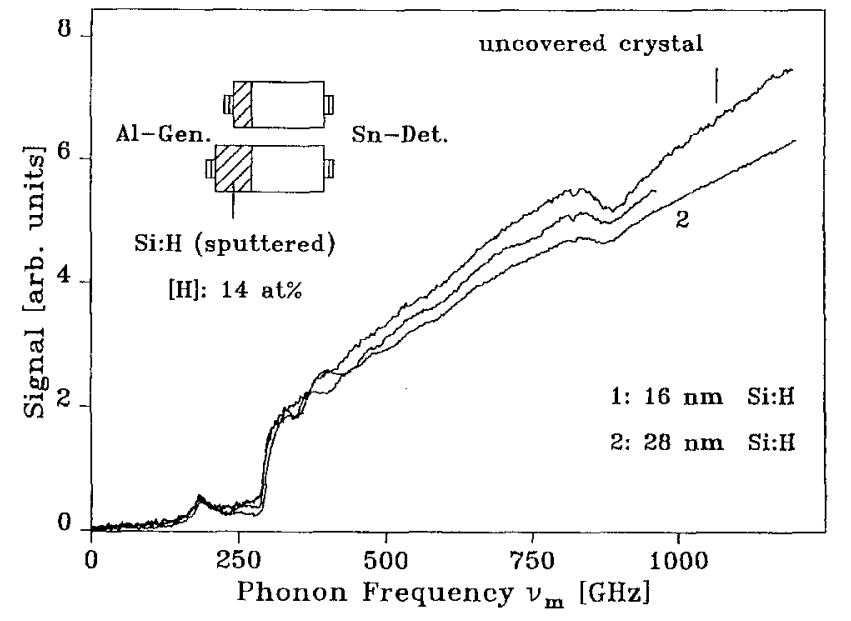

Fig. 10. Phonon transmission spectra of sputtered Si : $\mathbf{H}$ films with an hydrogen content of $14 \mathrm{at} \%$. The upper trace shows the spectrum of an uncovered carrier crystal

the $875 \mathrm{GHz}$ absorption structure is much enhanced compared to the measurements made with PECVD prepared films.

9. Heated $\mathrm{Si}: \mathrm{H}$ films. In the measurements described so far we could demonstrate that the $\mathrm{H}$-content of the amorphous films is an essential cause for the observed signal reduction and for the reduction of the depth of the $875 \mathrm{GHz}$ absorption structure in the phonon transmission spectra. In order to reveal more information in the question whether the molecularly bonded or the atomicaly bonded hydrogen gives the main contribution to the observed effects we performed measurements on heated $\mathrm{Si}: \mathrm{H}$ samples. The hydrogen release of hydrogenated amorpous Si by heating was investigated by Beyer [1]. The results show, that the molecularly bonded hydrogen escapes the film at temperatures of $300^{\circ} \mathrm{C}$, the atomicaly bonded hydrogen is released at $550^{\circ} \mathrm{C}$. The release of the atomicaly bonded hydrogen above $550^{\circ} \mathrm{C}$ was further confirmed by measurements of the spin density in heated $\mathrm{Si}: \mathrm{H}$ samples (Zellama [23]).

In our experiments we heated a $\mathrm{Si}: \mathrm{H}$ sample which was evaporated in a hydrogen atmosphere of $8 \cdot 10^{-5} \mathrm{mbar}$ in a quartz tube ( $\left.p \leq 10^{-2} \mathrm{mbar}\right)$ up to temperatures of $400^{\circ} \mathrm{C}$. In this first step the molecularly bonded hydrogen was released. To release the atomicaly bonded hydrogen we heated the sample in a second step up to a temperature of $550^{\circ} \mathrm{C}$.

In the upper traces of Fig. 11 we compare the signal course of a unheated sample with that of a sample which was heated at $400^{\circ} \mathrm{C}$. Both traces coincide after parallel shift. In the case of the sample which was heated at $550^{\circ} \mathrm{C}$ (lower traces) the unheated sample shows a higher signal reduction than the heated sample.

10. Nitrogen prepared semiconductors. In all measurements described so far the preparation process was done in an atmosphere of reactive gases. In this section we reveal the influence of an inertgas atmosphere on the phonon transmission spectra of amorphous films. 


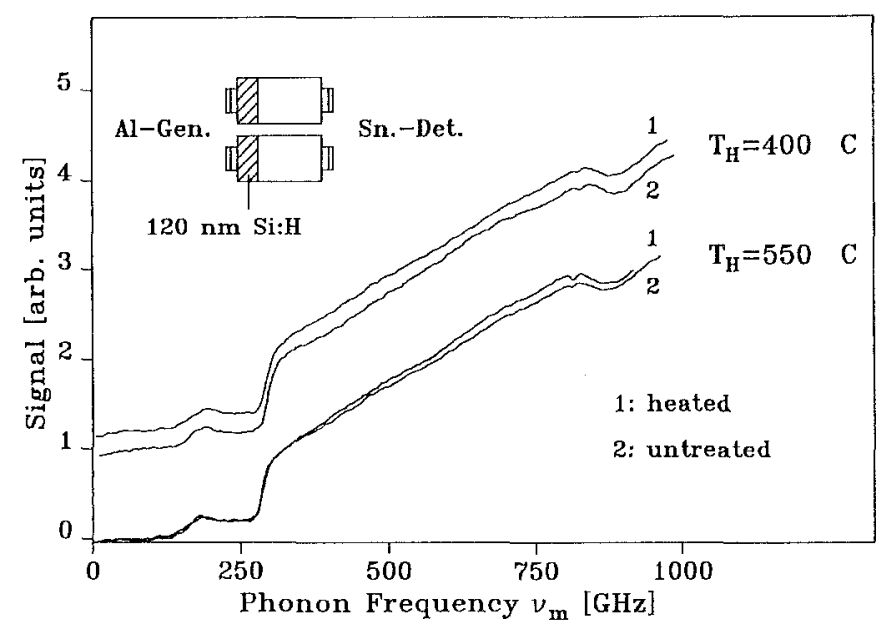

Fig. 11. Influence of the heating temperature on the phonon transmission spectrum of a Si: $\mathrm{H}$ film (film thickness $120 \mathrm{~nm}$ ). The films were prepared in a hydrogen atmosphere of $8 \cdot 10^{-5} \mathrm{mbar}$ and then heated. The measurements compare the spectrum of a unheated sample with that of samples which were heated at $400^{\circ} \mathrm{C}$ respective at $550^{\circ} \mathrm{C}$

In Fig. 12 we compare the phonon transmission spectra of pure $a$-Si and $a$-Ge films with that of nitrogen prepared films of same thickness. The $\mathrm{N}_{2}$ partial pressure was $2-4 \cdot 10^{-5}$ mbar during the evaporation process. In both cases the traces coincide within the entire frequency range. Concerning the phonon transmission spectra nitrogen prepared amorphous semiconductor films show the same behaviour than pure films. An influence of the perturbation gas could not be recognized.

\section{Pulse measurements}

In order to obtain more detailed information on the phonon scattering processes in $a$-Si which do obviously not affect the frequency resolved phonon transmission spectra we additionaly performed time resolved phonon transmission measurements with $a$-Si samples.

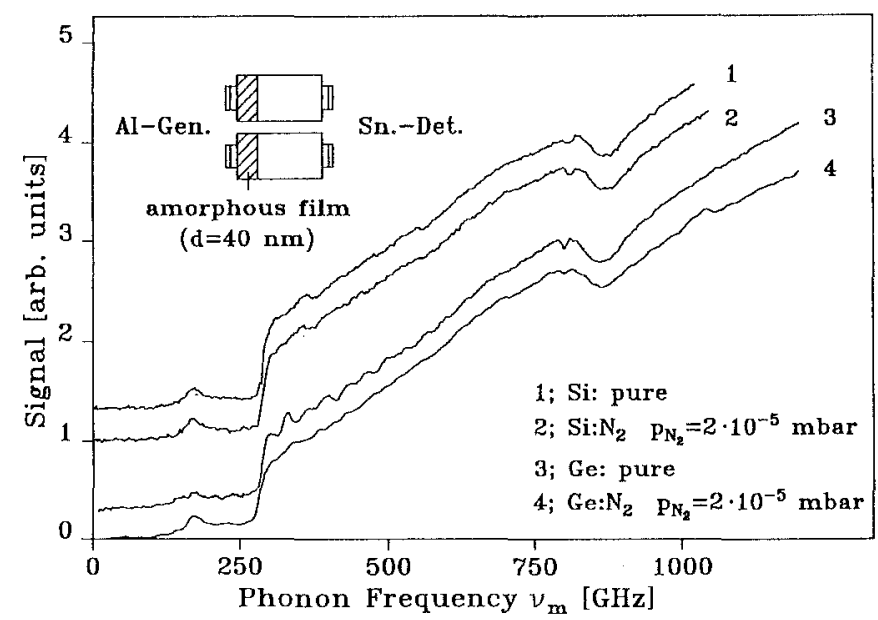

Fig. 12. Comparision of the phonon transmission spectra of nitrogen prepared semiconductor films with the spectra of films which were evaporated without residual gas

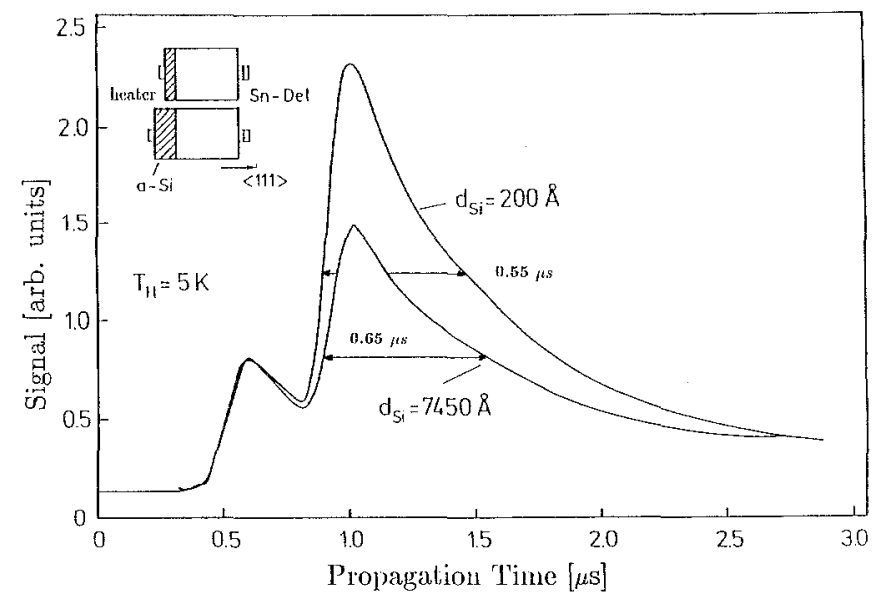

Fig. 13. Comparison of the pulse measurements with evaporated $a$-Si films. The film thicknesses were $20 \mathrm{~nm}$ and $750 \mathrm{~nm}$. The pulse width of the heater pulse was $50 \mathrm{~ns}$ and the heater temperature was $5 \mathrm{~K}$. The signal traces were adjusted to identical pulse height of the LA pulse

The carrier crystal in these measurements had a thickness of $4 \mathrm{~mm}$ and was only weakly doped with oxygen $\left(10^{16} \mathrm{~cm}^{-3}\right)$. These carrier substrates we selected for reducing the influence of the interstitial oxygen on the time resolved measurements. As phonon generator a constantan heater was evaporated on top of the amorphous film. For investigating the same detection range as in the frequency resolved phonon transmission measurements we used Sn tunnel junctions as phonon detectors.

The pulse measurements again were performed as "double crystal experiments". In order to have defined interface conditions we used crystals covered with different thicknesses of $a-\mathrm{Si}$. In such an arrangement the interface conditions between phonon generator and sample are equal in both experiments.

The two measurements of such a "double crystal experiment" were calibrated on identical pulse height of the LA pulse, because for comparison with the phonon transmission measurements the differences in the TA phonon system are important. This manner of calibration does not mean, that the intensity of the LA phonons is identical in both measurements.

In Fig. 13 the pulse measurement of an evaporated $20 \mathrm{~nm} a$-Si film is compared with that of a $750 \mathrm{~nm}$ sample. The pulse voltage was $1 \mathrm{~V}$. This relates to a heater temperature of $5 \mathrm{~K}$. The pulse width was $50 \mathrm{~ns}$. The time resolution in these experiments was $50 \mathrm{~ns}$.

In both signal courses a broadening of the phonon pulse can be recognized which scales with the film thickness. In the case of the $20 \mathrm{~nm}$ sample the pulse width at the half value is $0.55 \mu \mathrm{s}$ and increases to $0.65 \mu \mathrm{s}$ at the $750 \mathrm{~nm}$ sample.

The signal intensity of the TA pulse decreases (relative to the LA pulse) with increasing film thickness.

A thickness dependent delay of the phonon pulses could not be recognized within the resolution range. 


\section{Discussion}

\section{A. General considerations}

In our experiments the phonon generator was prepared on top of the amorphous film. In this arrangement the phonons must transmit the amorphous film before they can interact with the carrier crystal. In the amorphous film the spectrum emitted by the generator junction can be modified by elastic or inelastic phonon scattering processes.

1. Elastic scattering processes. Elastic scattering processes can only change the distribution of the emission directions of the emitted phonons. They do not change their energy distribution. This change of the phonon emission directions results in a modification of the geometrical radiation conditions of the phonons at the filmsubstrate surface. Further the phonons can be scattered back into the generator junction or in the surrounding He-bath.

In superconducting tunnel junctions phonons are generated by relaxation processes of quasiparticles. These quasiparticles are excited in both films by tunneling processes. Because of their high velocity (Fermi velocity) the quasiparticles in the generator junction are scattered with high probability before they can relax under phonon emission. This leads to a statistical distribution of the phonon emission directions at the generator surface. The resulting emission distribution can be described by the Lambert's cosine law. The addition of elastic scattering processes in an amorphous film between generator junction and carrier crystal may not change these emission conditions.

Elastic scattering processes we further expect to lead to a broadening of the "hot spot" from which the phonons are mainly emitted. In the case of an almost isotropic propagation of the phonons in the amorphous film, this broadening is only of the same order as the film thickness (some $10 \mathrm{~nm}$ ). With a detector area of $1 \mathrm{~mm}^{2}$ this broadening can be expected not to affect the intensity detected by the $\mathrm{Sn}$ junction.

In contrast to the emission conditions at the filmcrystal boundary the backscattering of phonons into the generator junction or the surrounding He bath may induce a strong influence on the signal course. Because elastic scattering processes always increase with rising frequency such elastic backscattering must lead to an increasing signal reduction with increasing phonon frequency. Such signal reduction is only possible, if the phonon mean free path for elastic scattering $\left(\Lambda^{\mathrm{el}}\right)$ is much smaller than the film thickness $d$. In the case of "weak" elastic scattering $\left(\Lambda^{\mathrm{el}}>d\right)$ the loss of energy flux by backscattering does not give an influence on the detector signal.

2. Inelastic scattering processes. In contrast to the eleastic scattering processes the inelastic scattering processes change the energy distribution of the phonon spectrum. In the presence of inelastic phonon scattering in the amorphous film between generator junction and carrier crystal the scattering processes result in a modified phonon spec- trum $\dot{N}_{\mathrm{Ph}}^{\mathrm{in}}(\Omega, V)$ at the film-substrate boundary. This modified spectrum leads to modifications of the signal course of the phonon transmission spectrum.

Due to the energy loss of the phonons some phonons can not be detected, because their energy is below the detection threshold. In the energy counting range of the detector [6] the energy loss results in a reduced signal participation of the scattered phonons. Therefore inelastic phonon scattering in the film always leads to a signal reduction in the phonon transmission spectrum. This signal reduction increases with increasing number of scattering processes i.e. with the film thickness.

A strong elastic backscattering of phonons into the generator junction may also lead to a signal reduction in the transmission spectrum. This is possible due to the energy loss of the backscattered phonons by pair breaking effects in the generator junction. This generator induced inelastic scattering must be separated from the inelastic scattering in the film. One possibility to do this are transmission measurements on samples with the amorphous film below the detector junction. Due to the thickness of the carrier crystal in this arrangement a generator induced influence can be excluded. The signal reduction in this case is of the same magnitude as for an equivalent sample with the amorphous film below the generator junction so that we can ascribe the signal reduction to inelastic phonon scattering processes in the amorphous film.

\section{B. Discussion of the measurements}

1. Pure amorphous semiconductors. The phonon transmission measurements on pure amorphous semiconductor films do not show any influence of the amorphous film on the signal course (Fig. 2 and 3). In the case of $a$ $\mathrm{Si}$ as well as in the case of $a-\mathrm{Ge}$ the signal traces were identical to those of an uncovered carrier crystal. For this we can say that in pure amorphous semiconductor films either a ballistic propagation of phonons or a weak elastic phonon scattering exists. In this question pulse measurements can give additional information.

The pulse measurements described above show that there is no ballistic propagation of phonons in the pure amorphous semiconductors (Fig. 13). This can be seen by the enhanced half width of the pulse and by the thickness dependence of the reduction of the TA pulse (in relation to the LA pulse). The obviously higher scattering probability of the TA phonons is typical for scattering processes by structural inhomogeneties. In these scattering processes the scattering probability decreases with increasing sound velocity (Klemens [16]). In contrast to this in scattering processes at localised scattering centers which can be described in the elastic dipole model (Nowick, Heller [19]), the scattering probability increases with the sound velocity, because in this case the interaction energy between the scattering center and the phonon field is proportional to the elastic constants and therefore to the square of the sound velocity.

Scattering processes at localised scattering centers should therefore reduce the LA pulse more than the TA pulse. Thus the observed behaviour gives evidence on 
elastic scattering processes at structural inhomogenities in the pure amorphous semiconductors.

Such elastic phonon scattering does not affect the phonon transmission measurements, if the phonon mean free path is higher than the film thickness. We therefore can give a lower limit of the phonon mean free path by the value of the investigated film thicknesses (order of $10 \mathrm{~nm}$ ).

2. $\mathrm{SiO}_{2}$-films. In contrast to the pure amorphous semiconductors the phonon transmission measurements on $\mathrm{SiO}_{2}$ films show a strong influence of the amorphous film on the signal course. As discussed above the observed signal modification can be explained by inelastic phonon scattering in the amorphous film or by a strong backscattering of the phonons into the generator junction. A decision of this question is possible by additional measurements at samples with the amorphous film between carrier crystal and detector junction. This measurement (Fig. 5) shows that the signal reduction is of the same magnitude as in measurements with the amorphous film below the generator junction. This confirms that the signal reduction in the phonon transmission measurements of $\mathrm{SiO}_{2}$ films is due to inelastic phonon scattering processes in the film.

In the measurement with the $\mathrm{SiO}_{2}$ film below the detector junction the absorption depth of the $875 \mathrm{GHz}$ resonance structure seems to be not affected by the amorphous film. This behaviour can be explained as follows. A disadvantage of measurements with the amorphous film between carrier crystal and detector junction is, that the phonon spectrum modified by the inelastic scattering processes does not interact with the vibrational resonance of the interstitial oxygen in the carrier crystal. That means that the monochromatic phonon line of the generator spectrum interacts in its full intensity with this oxygen resonance. The respective phonons are scattered out of the detection channel before they can interact with the amorphous film. Consequently the resonance structure of the carrier crystal gives a full "image" in the phonon transmission spectrum. In contrast, when the amorphous film is prepared between the generator junction and the carrier crystal the intensity of the phonon line at $875 \mathrm{GHz}$ is reduced by scattering processes in the film and the information about the reduction of the intensity of the monochromatic phonon line at $875 \mathrm{GHz}$ is not lost.

The measurements on thermally oxydized films show the same behaviour as those on evaporated $\mathrm{SiO}_{2}$ films. Nevertheless the signal reduction in thermally oxydized films is less in comparison with evaporated films of the same thickness.

In both systems the gradient in the signal course of the transmission spectrum increases in the frequency range above $870-880 \mathrm{GHz}$. This behaviour is independent of the film thickness. The enlargement of the gradient in the signal course above $870-880 \mathrm{GHz}$ corresponds to a reduction of the scattering strength of the inelastic scattering processes. Therefore the scattering strength seems to have a maximum in the frequency range of $870-880 \mathrm{GHz}$. This behaviour is typical for a "linear Raman effect" with a resonance frequency at $870-880 \mathrm{GHz}$. This influence will be discussed in a further publication. All other scat- tering processes characterized by an increasing scattering strength with phonon frequency (such as the "quadratic Raman effect" proposed by Leadbetter [17]) must lead to a monotonically decreasing gradient in the phonon transmission spectrum.

The film induced reduction of the absorption depth of the $875 \mathrm{GHz}$ resonance structure of the vibrational excitation of the interstitial oxygen in the carrier crystal gives the possibility to evaluate the phonon mean free path in the amorphous film. In a simple model we can assume the intensity of the monochromatic phonon line at $875 \mathrm{GHz}$ is reduced exponentialy by the amorphous film

$I(875 \mathrm{GHz})=I_{0}(875 \mathrm{GHz}) \exp (-d / \Lambda(875 \mathrm{GHz}))$

In highly doped carrier crystals the absorption depth corresponds to the intensity of the phonon line at $875 \mathrm{GHz}$. With known thickness of the amorphous film the phonon mean free path $\Lambda(875 \mathrm{GHz})$ can be evaluated by examination of the absorption depth of the $875 \mathrm{GHz}$ resonance structure. For determination of the absorption depth we used the simplified procedure shown in Fig. 4. The related values for evaporated $\mathrm{SiO}_{2}$ and thermal oxide are shown in Table 2.

The larger value of the phonon mean free path in thermally oxidized films indicates a dependence of the phonon scattering on the structure of the amorphous film. Thermally oxidized films have a much denser structure than evaporated $\mathrm{SiO}_{2}$ films. Correspondingly all scattering processes dependent on structure are less pronounced. Therefore in amorphous $\mathrm{SiO}_{2}$ an additional elastic scattering at structural inhomogenities appears to be possible. Such structural inhomogenities might be the so called voids, which were discussed by Gibson and Dong [9].

3. Oxygen prepared films. The phonon transmission measurements on semiconductor films, prepared by oxygen background evaporation show the same characteristics as $\mathrm{SiO}_{2}$. In the oxygen prepared films there seems to exist the same inelastic scattering processes than in $\mathrm{SiO}_{2}$.

Table 2. Phonon mean free path of phonons at $875 \mathrm{GHz}$ in evaporated $\mathrm{SiO}_{2}$ and thermal oxide. The values are determined by the reduction of the $875 \mathrm{GHz}$ resonance structure of the carrier crystal

\begin{tabular}{lc}
\hline & \multicolumn{1}{c}{$\frac{\Lambda(875 \mathrm{GHz})[\AA]}{50-100}$} \\
Evaporated $\mathrm{SiO}_{2}$ & $200-350$
\end{tabular}

Table 3. Phonon mean free path of phonons at $875 \mathrm{GHz}$ in oxigen prepared amorphous semiconductors. The values are determined by the reduction of the $875 \mathrm{GHz}$ resonance structure of the carrier crystal

\begin{tabular}{lll}
\hline & Pertubation gas pressure [mbar] & $\Lambda(875 \mathrm{GHz})[\AA]$ \\
\hline $\mathrm{Si}: \mathrm{O}_{2}$ & $4 \cdot 10^{-5}$ & $500-600$ \\
$\mathrm{Ge}: \mathrm{O}_{2}$ & $4 \cdot 10^{-5}$ & $700-800$ \\
\hline
\end{tabular}


The thickness dependence of the reduction of the absorption depth of the $875 \mathrm{GHz}$ resonance structure can be used to examine the phonon mean free path $\Lambda(875 \mathrm{GHz})$ in the discussed way. The values are collected in Table 3.

The inelastic scattering processes are present in oxygen prepared $a$ - $\mathrm{Si}$ as well as in oxygen prepared $a$-Ge. But in both cases the scattering strength is less than in $\mathrm{SiO}_{2}$. Apparently the centers of the inelastic phonon scattering correspond to oxygen imbedded in the amorphous network. Due to the high oxygen content in $a-\mathrm{SiO}_{2}$ the scattering strength is much larger than in oxygen prepared films.

In this consideration the structure of the amorphous network has a strong influence on the resulting scattering strength of the inelastic scattering processes. First the morphology of the film determines the concentration of the included oxygen in the film. Further the structure of the network leads to elastic scattering processes enlarging the propagation time of the phonons through the network. This results in an increasing number of interactions of the phonons with the centers of the inelastic scattering processes.

In this context the enhanced phonon mean free path in $\mathrm{Ge}: \mathrm{O}_{2}$ compared with that in $\mathrm{Si}: \mathrm{O}_{2}$ (Table 3) can be interpreted in terms of the more homogenous network structure of Ge.

4. Hydrogen prepared films. The phonon transmission spectra of hydrogen prepared Si films also show characteristics of inelastic phonon scattering. The phonon mean free path $\Lambda(875 \mathrm{GHz})$ can be examined by the thickness dependence of the depth of the $875 \mathrm{GHz} a b-$ sorption line (Table 4). The scattering strength increases with the hydrogen content of the film.

These results show, that hydrogen as well as oxygen leads to inelastic phonon scattering in the amorphous system. The question whether the molecularly or atomicaly bonded hydrogen is responsible for the phonon scattering can be decided by the experiments on heated samples. In these experiments a significant reduction of the scattering strength can be recognized in samples which were heated to temperatures at which the atomicaly bonded hydrogen diffuses out of the system. In contrast the release of the molecularly bonded hydrogen does not affect the spectral signal.

5. Nitrogen prepared films. The phonon spectroscopy measurements discussed so far show that chemically bounded gas atoms in the sample result in an inelastic phonon scattering in the amorphous film. This interpre-

Table 4. Phonon mean free path of phonons at $875 \mathrm{GHz}$ in hydrogen prepared Si films. The values are determined by the reduction of the $875 \mathrm{GHz}$ resonance structure of the carrier crystal

\begin{tabular}{lcc} 
& H-content $[\%]$ & \multicolumn{1}{c}{$A(875 \mathrm{GHz})[\AA]$} \\
\hline Si (evaporated) & $<10 \%$ & $2400-2700$ \\
Si (PECVD) & ca. $10 \%$ & $900-1200$ \\
Si (sputtered) & ca. $14 \%$ & $300-600$ \\
\hline
\end{tabular}

tation can additionaly be checked by the measurements on films prepared in a nitrogen atmosphere. Nitrogen does not change the chemical composition of the film but influences the network structure of the film. This is an effect of the reduced surface mobility of the absorbed particles during the evaporation process. Figure 12 shows that the preparation of the film in a $\mathrm{N}_{2}$ atmosphere does not affect the phonon transmission spectra.

\section{Conclusion}

In this work we investigated the phonon scattering in amorphous films with the use of phonon spectroscopy measurements in the frequency range from $285 \mathrm{GHz}$ to $1 \mathrm{THz}$. Our measurements include the pure amorphous semiconductors $a$-Si and $a$-Ge as well as $a-\mathrm{SiO}_{2}$ and hydrogen doped $a$-Si: $\mathrm{H}$.

The amorphous films were prepared in different thicknesses on identical carrier crystals. The film thickness ranged in the order of several $10 \mathrm{~nm}$.

The phonon transmission spectra of films in this thickness range show in the lower frequency range resonant structures due to thickness resonances in the film. These were used to determine the sound velocity in the amorphous materials.

The further analysis of the phonon transmission spectra of the films show only elastic scattering processes in the pure amorphous semiconductors. In contrast to this in $a-\mathrm{SiO}_{2}$ an inelastic contribution was identified. This inelastic contribution leads to signal modifications in the phonon transmission spectra which could be interpreted by inelastic scattering processes induced by a "linear Raman effect". Similiar signal modifications could also be observed in amorphous semiconductors which were prepared in an oxygen or hydrogen atmosphere. In $a$ $\mathrm{Si}: \mathrm{H}$ the atomicaly bounded hydrogen was identified as center of the inelastic scattering processes.

We extend our gratitude to Dr. K. Laßmann for numerous stimulating and fruitful discussions. We wish to thank the "Max Planck Institut für Festkörperphysik" in Stuttgart and the "Institut für Physikalische Elektronik" at the University of Stuttgart for preparing a large number of samples. The financial support by the "Stiftung Volkswagenwerk" is gratefully acknowledged.

\section{References}

1. Beyer, W.: J. Non-Cryst. Solids 97/98, 1027 (1987)

2. Brodsky, M.H., Kaplan, D., Ziegler, J.F.: Appl. Phys. Lett. 21, 305 (1972)

3. Brodsky, M.H., Frisch, M.A., Ziegler, J.F.: Appl. Phys. Lett. 30, 561 (1977)

4. Dietsche, W., Kinder, H.: Phys. Rev. Lett. 43, 1413 (1979)

5. Eisenmenger, W., Dayem, A.H.: Phys. Rev. Lett. 18, 125 (1967)

6. Eisenmenger, W.: Physical acoustics, Vol. XII. New York: Academic Press 1976

7. Forkel, W.: Dissertation. Universität Stuttgart 1977: (unpublished)

8. Forkel, W., Welte, M., Eisenmenger, W.: Phys. Rev. Lett. 31, 215 (1973)

9. Gibson, J.M., Dong, D.W.: J. Electrochem. Soc. 127, 2722 (1980)

10. Graczyk, J.F.: Phys. Status Solidi (a) 55, 231 (1979)

11. Graebner, J.E. et al.: Phys. Rev. B29, 3744 (1984) 
12. Gutsow, I.: Z. Phys. Chem. 221, 153 (1962)

13. Irene, E.A. et al.: J. Electrochem. Soc. 127, 396 (1980)

14. Kinder, H.: Phys. Rev. Lett. 28, 1564 (1972)

15. King, C.N., Phillips, W.A.: Phys. Rev. Lett. 32, 358 (1974)

16. Klemens, P.G.: Proc. Phys. Soc. 68, 12 A, 73 (1955)

17. Leadbetter, A.J. et al.: J. Phys. 38, 95 (1977)

18. Mertig, M. et al.: Solid State Commun. 49, 369 (1984)
19. Nowick, A.S., Heller, W.R.: Adv. Phys. 12, 251 (1963)

20. Pompe, G., Hegenbarth, E.: Phys. Status Solidi (b) 147, 103 (1988)

21. Shiojiri, M. et al.: Jpn. J. Appl. Phys. 18, 1931 (1979)

22. Wolter, J., Horstmann, R.E.: Solid State Commun. 37, 171 (1981)

23. Zellama, K., Germain, P.: Phys. Rev. B23, 6648 (1981) 